\title{
Acrosswind Response of Reinforced Concrete Chimneys
}

\author{
C-M. Cheng ${ }^{a}$ and Ahsan Kareem ${ }^{b}$ \\ aTamkang University, Tamsu, Taipei Hsien, Taiwan 25137, Republic of China \\ bepartment of Civil Engineering, University of Notre Dame, Notre Dame, Indiana \\ 46556-0767
}

\begin{abstract}
The acrosswind response of isolated reinforced concrete chimneys of circular cross-section is studied using wind tunnel tests, full-scale measurements and response predictions based on semi-empirical methods. Three chimneys with available full-scale response observations were selected for this study to compare their measured and predicted responses. The wind tunnel experiments involved measurements of unsteady aerodynamic loads on rigid models of circular cross-section and aeroelastic response of scale models of full-scale chimenys. Tests were conducted initially on smooth surface models, which were repeated with artificially roughened surface. Attachment of discrete two dimensional surface roughness helps to simulate artificially flow field features past a cylinder that represent the characteristics of high Reynolds number flows. Utilizing this roughness configuration permits response prediction of chimneys in boundary layer wind tunnels with a good agreement with the observed full-scale response.
\end{abstract}

\section{INTRODUCTION}

The acrosswind (lift) force is recognized as a significant source of wind excited motion of tall chimneys. Due to complexity of the problem, no analytical model based on an understanding of the flow field around circular chimneys has been established that might satisfactorily predict the aerodynamic response of chimneys in atmospheric boundry layer flows. From analytical point of view, solution of coupled fluid and structural motions is involved which is presently mathematically intractable. However, recent advances in computational fluid dynamics may provide a solution based on coupled fluid-structure motion. Presently, semi-empirical models have been proposed in lieu of the solutions of the coupled system of equations. The first group of such techniques falls into the category of equivalent static approaches. The second category is based on the extension of forced vibration theory with the motion dependent forces represented by a nonlinear aerodynamic damping term. The experimental observations of the phenomenon have suggested that an oscillating cylinder/wake combination possesses the characteristics of a nonlinear oscillator. This has prompted the use of a Van der Pol oscillator-type models to represent this behavior.

The inability to simulate high Reynolds number flows in boundary layer type tunnels has impaired the development of predictive approaches based on experimental methods. The only studies involving flow around circular cylinders with measurements made at Reynolds numbers comparable to those typical of full-scale 
structures in atmospheric boundary layers are those of long, stationary, circular cylinders (two-dimensional) in uniform flows at low levels of turbulence in aeronautical type wind tunnels. However, predictive models based on these studies would entail restrictions or biases. Indeed rather than being uniform, the atmospheric flows are characterized by height dependent mean velocities and turbulence. The vortex shedding from a circular cylinder in shear flows occurs in spanwise cells of different frequencies and correlation lengths. The presence of turbulence in the approach flow acts as a modifier of the vortex shedding process that depends on the length scale and the intensity of turbulence. An additional difference between the conditions of the previously mentioned experiments and full-scale chimneys is the presence of a threedimensional flow field due to the flow over the free-end of the chimneys.

This paper reports some of the results of a study undertaken to increase our understanding of the wind-structure interactions involving finite height cylinders in atmospheric flows. This would assist in predicting the dynamic wind load effects on chimneys. An isolated and an array of finite height cylinders were utilized to map the pressure and force fields in simulated flows in a boundary layer tunnel. For a single cylinder, the surface of the model was provided with various types and sizes of discrete and homogenous rouginness elements to achieve an optimal surface roughness to create flow features past the cylinder similar to the high Reynolds numbers of full-scale conditions. Dynamic models of isolated circular cylinders with different mass damping parameters (Scruton number) were used to verify the acrosswind force measurements by comparing response estimates based on the measured force and experimentally observed response of cylinders. These measurements also provided useful information on the motion dependent forces on cylinders. Aeroelastic model tests were conducted for three chimneys for which full-scale measurement were available (Muller and Niesser, 1976; Christensen and Askegaard, 1978; Hansen, 1981 and Melbourne, et al, 1983). These chimneys are 130m located in Denmark, $180 \mathrm{~m}$ chimney in Germany and $265 \mathrm{~m}$ chimney located in Australia. Additional aeroelastic tests were also conducted in a boundary layer wind tunnel in R.O.C. The measured full-scale response of chimneys was compared to the response of aeroelastic models, to response predictions based on measured force spectra in artificially simulated high Reynolds number flows and to the predictions based on various semi-empirical procedures available in the literature.

The verification or validation of experimental procedures or theoretical methods lies in the comparison with the observed full-scale data. Despite the uncertainties associated with the full-scale data, e.g., limited data, variability of the estimation of freestream wind conditions, this information offers at present the only possible means of demonstrating the validation of the laboratory simulations or theoretical predictions.

\section{EXPERIMENTAL}

Most of the experiments were conducted in boundary layer wind tunnel laboratory at the University of Houston. Additional aeroelastic tests were made at the Academia Sinica's boundary layer wind tunnel in R.O.C. The atmospheric boundary layers were generated by the natural action of the surface roughness added on the tunnel floor and upstream spires. Two different approach flow boundary layers were used that represent open country and urban flow conditions. Three different types of models were used that include rigid plexiglass models of uniform circular cross-section to monitor point and area-averaged pressures, and aeroelastic models of prototype 
chimneys. The point pressure measurement model was utilized to configure optimal distribution of surface roughness to simulate artificially high Reynolds number flow past cylinders. The averaged pressure measurements using pnsumatic averaging manifolds were utilized to measure unsteady aerodynamic folces at five levels. A circular cross-section manifold tubing system was utilized in this study. Each measurement level was divided into two semi-circles. Each semi-circle had seven pressure taps which were weighted such that the manifolded pressure would represent resultant lateral force. The two manifolds facing opposite sides were differenced by an electronic pressure transducer to obtain lateral loads at that level. Five levels on the cylinder were simultaneously monitored. A covariance integration scheme was utilized to determine the mode generalized loading. These measurements in conjunction with a random vibration based analysis provided convenient response predictions. Details of the pneumatic averaging manifolds and their dynamic calibration procedure are described elsewhere (Kareem \& Cheng, 1984).

A spine-type construction was utilized to develop the first set of aeroelastic models of $130 \mathrm{~m}$ and $180 \mathrm{~m}$ chimneys (Christensen, 1978; Mueller \& Niesser, 1976 and Kareem and Cheng). The spine model consisted of a continuous aluminum tube to provide the proper stiffness and a polyurethane surface shell to model the actual structural envelope. The second set of models were made for $130 \mathrm{~m}$ and $265 \mathrm{chim}$ neys (Christensen, 1978 and Melbourne, 1983). These models were made out of Devcon. The flow conditions at these chimney sites were appropriately modelled to represent the reported flow field characteristics, at their respective sites.

\section{SIMULATION OF HIGH REYNOLDS NUMBER FLOW}

The shortcoming of typical boundary layer wind tunnels in simulating high Reynolds number flow can be overcome by artificially modifying the flow field around the model. This can be accomplished by employing roughness on the model surface (Schlichting (1979), Achenbach (1981), Batham (1973), Farell and Guven (1976), Nakamura and Tomonrai (1982), and Szechenyi (1975)). Most of these experiments were conducted at Reynolds number in the range between high $10^{4} \sim$ low $10^{5}$. The most effective simulation of super critical Reynolds number flow was achieved at $R e$ $\cong 10^{5}$ regardless of the type of roughness. In contrast to the preceding measurements that utilized uniform surface roughness, Naumann and Ouadfleig (1972) applied a pair of two dimensional roughness (wires) on the surface of a circular cylinder and succeeded in simulating supercritical Reynolds number flow features at approach flow Reynolds number as low as $10^{5}$.

In the first phase of this study, various types of surface roughness elements were installed on finite height models of circular cross section in an effort to artificially simulate the high Reynolds number flows around cylinders immersed in boundary layer flows. For proper scaling of turbulence in the boundary layer wind tunnels used here, the Reynolds number based on the cylinder diameter were limited to between $10^{3}$ and $10^{4}$. Among various surface roughness types investigated in this study, the two dimensional roughness elements resulted in flow simulation that closely represented the high Reynolds number flows. The optimal configuration in this study at both wind tunnel laboratories was found to be a pair of wires with diameter $d / D=0.0065$ at \pm $65^{\circ}$ from the front stagnation line and another pair with ciameter $\mathrm{d} / \mathrm{D}=0.02$ at $\pm 115^{\circ}$ ( $d=$ wire diameter and $D=$ cylinder diameter). The first pair of wires are intended to perturb the laminar flow in the surface boundary layer and cause transition to turbulent flow, whereas, the second pair causes flow separation at the desired location. In 
Fig. 1, the mean pressure distribution around the cylinder with and without roughness wires is presented. The model 3-II represents continuous wire roughness, whereas the model 3-III has 3 inches long pieces of roughness of same cross-section, but installed in a staggered configuration such that at the top they start with same orientation and $a \pm 5$ degrees shift is added to alternative lengths in order to examine the influence of two-dimensional effects (Kareem and Cheng, 1984). The pressure distribution for a smooth cylinder represents characteristics of subcritical Reynolds number. Whereas, for roughned cylinders the pressure distribution is similar to supercritical Reynolds number flows as described typically by full-scale data. A minimum Reynolds number of $2.5 \times 10^{4}$ is neecied to attain the above flow features.

The roughness configuration described above was placed on a model fitted with pneumatic averaging manifolds for measuring unsteady forces at different levels on the model. The multiple-level data from model study with roughness was utilized to develop mode generalized acrosswind force spectra for subsequent response analysis of structures with circular cross-section (Kareem and Cheng, 1984).

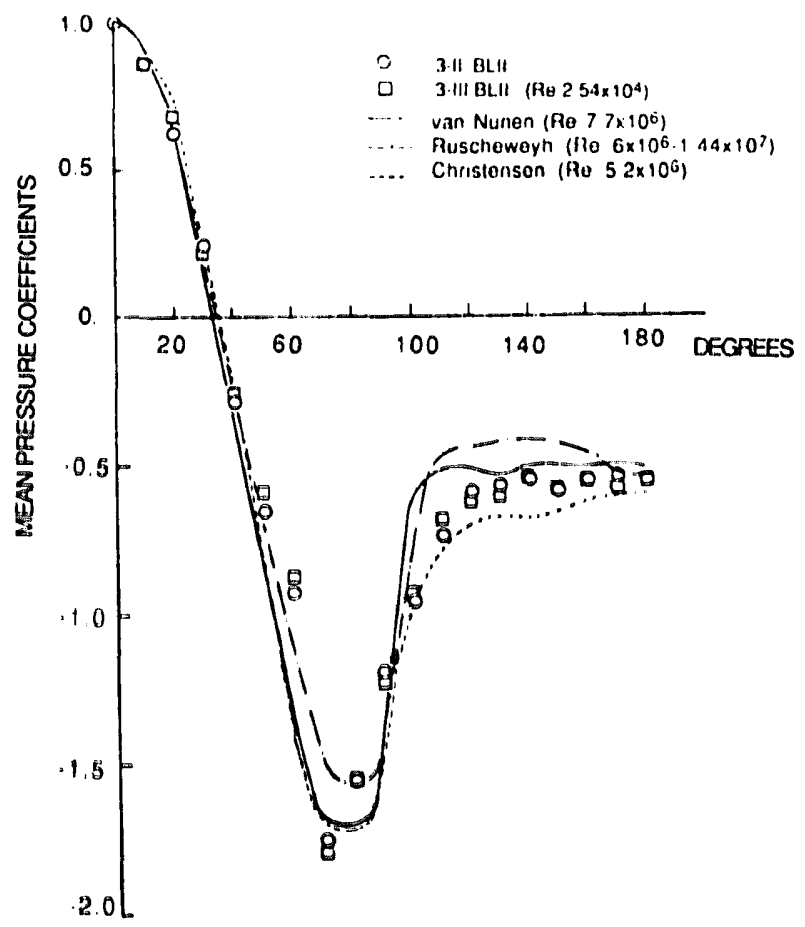

Figure 1. Mean pressure distribution on smooth and rough (wires) cylinders.

\section{CHIMNEY RESPONSE PREDICTION}

The acrosswind response of chimneys can be obtained by one of the following procedures: i) Response analysis based on measured force spectra; ii) Semi-analytical and empirical procedures; and iii) Response prediction based on aeroelastic models. These procedures have been employed to predict response of three chimneys for which full-scale response data are available (Muller and Niesser, 1976; Christenson and Askegaard, 1978 and Melbourne et al., 1983). Details are given below. 


\subsection{Response Analysis Based on Measured Force Spectra}

The response estimates for the three chimneys were made based on random vibration theory utilizing measured force spectra on a uniform circular cross-section model with surface roughness. The measured spectra could not be utilized in a straightfonward manner since the chimneys being studied have cross-section that tapers along the height as apposed to being uniform. In view of the small taper, an equivalent diameter was used for analysis. Implications arising from this idealization and the influence of aspect ratio are insignificant for these examples and a detailed discussion is provided elsewhere (Kareem and Cheng, 1984). The basic structural features were used to formulate the mass and stiffness matrices which were then employed to calculate the natural frequencies and mode shapes. The error in the estimation of fundamental frequencies compared to the reported values was within 2.5 percent. The mode-generalized loading spectrum was modified to account for each chimney mode shape (Kareem, 1982,1984). The rms acrosswind response estimates were made, utilizing white noise idealization of the forcing function in conjunction with the Residue Theorem, (e.g., Kareem, 1987)

$$
\sigma(z)=\left[\sum_{n=1}^{N} \frac{\phi_{n}^{2}(z) \pi f_{n} S_{F}\left(f_{n}\right)}{4 \xi_{n}\left(2 \pi f_{n}\right)^{4} M_{n}^{2}}\right]^{1 / 2}
$$

in which $S_{F}\left(f_{n}\right)=$ measured generalized acrosswind force spectrum evaluated at $f_{n}, f_{n}$ and $\xi_{n}=$ natural frequency and damping in the nth mode, respectively $M_{n}=$ modegeneralized mass, $\phi_{n}(z)=n$th mode shape, $N=$ total number of modes and $\sigma(z)=$ rms displacement at level $\mathbf{z}$.

\subsection{Semi-Analytical and Empirical Procedures}

Methods for predicting the response of chimneys given by Vickery and Basu (1983a), ESDU (1978) and equivalent static methods reported by Vickery and Basu (1983b) and van Koten (1986) are examined here. A summary of these procedures along with the information concerning their parameters is detailed in Simiu and Scanlar (1986).

The vortex induced forces on a structure may be expressed in terms of the forces on a stationary cylinder and those induced by structural motion. The forces on a stationary cylinder as stated above may be measured by a number of experimental techniques, e.g., pneumatic averaging. The force spectrum may be expressed in terms of a covariance integration scheme (Vickery and Clark, (1972), Kareem, (1984)). Based on experimental measurements Vickery and Clark (1972) developed a model to predict the response of chimneys to vortex induced excitation. Once the amplitude of the motion becomes large, the models based on the flow field around a stationary cylinder no longer represent the acrosswind force. An additional force component which is associated with the motion of a structure must be included Kareem, 1981 (Vickery and Basu, 1983a).

The method described in Engineering Science Data Unit item number 78006 (1978) for the prediction of the acrosswind response of an isolated circular crosssection stack may be separated into broad-band and narrow-band regimes. For smaller amplitudes the response is more likely to be described by a broad-band process, whereas for sufficiently large amplitudes of oscillation the response almost approaches a sinusoidal form. A detailed step-by-step tabulated procedure to estimate the acrosswind response of isolated circular cylindrical structures due to vortex shedding is available in ESDU (1978). In this procedure, many flow induced parame- 

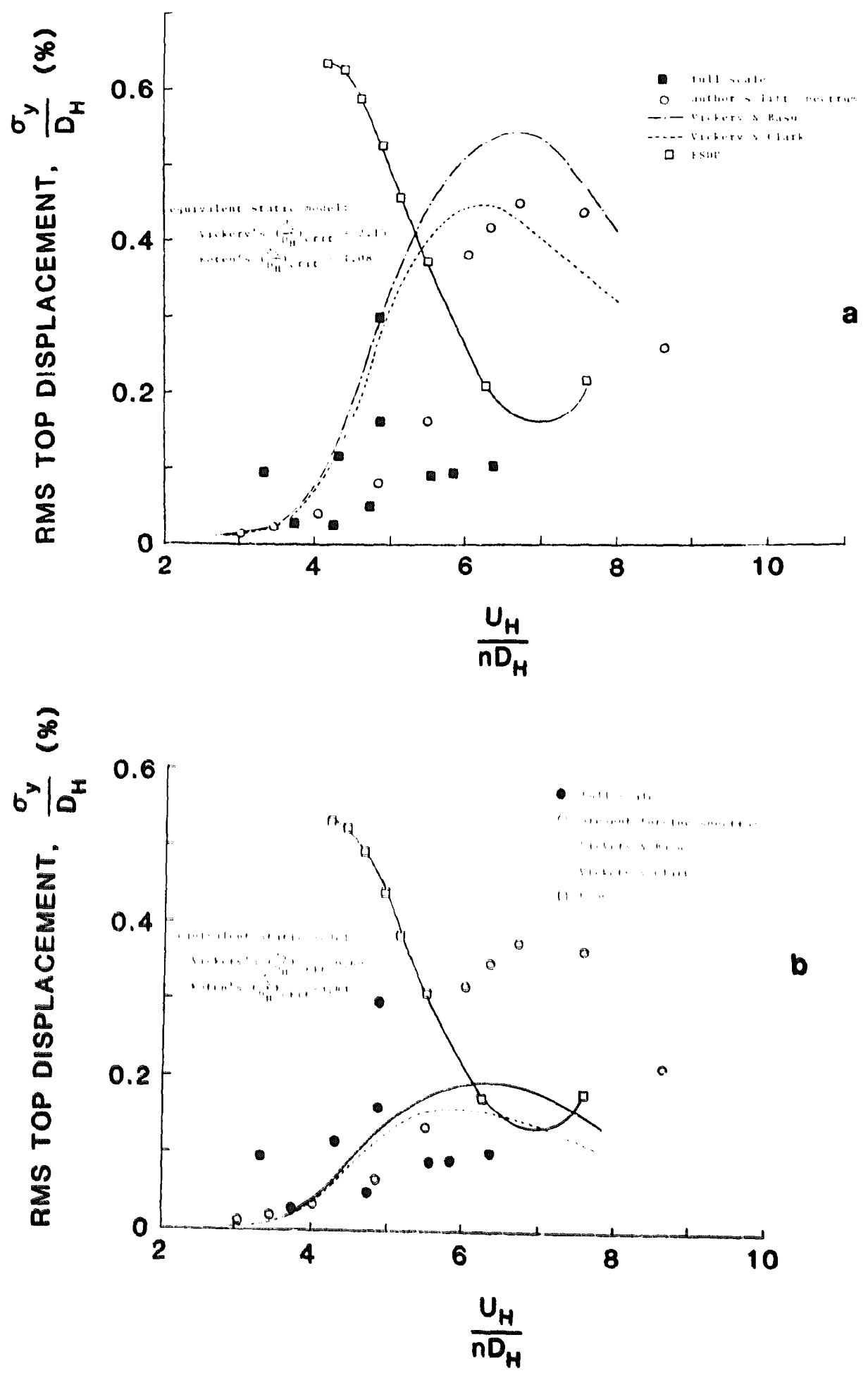

Figure 2. a) Comparison of predicted response with full-scale measurements of $130 \mathrm{~m}$ chimney; b) prediction based on adjusted model parameters. 
ters such as the Strouhal number, acrosswind force coefficient, and correlation length are initially obtained from data pertaining to two-dimensional flow conditions. Subsequently, the effects of free-stream turbulence, structural surface roughness and the aspect ratio are included using empirical data. The motion induced forces are derived on the premise that the oscillatory behavior of the chimney causes the wake to act a nonlinear, self-excited oscillator which produces an amplification of acrosswind forces. Empirical correlations for parameters are established from data for both stationary and oscillatory cylinders. Despite the adjustments for turbulence, surface roughness, and oscillation amplitude of motion through empirical methods, the uncertainty in this approach remains high. An additional shortcoming of this procedure is tha' it lacks a natural transition between the two regions which lends arbitrariness to this approach (Vickery, Basu, 1983a).

Alternatively, an equivalent static approach that converts the complex aerodynamic loading into an equivalent static load may be utilized. van Koten (1979) proposed an equivalent static model that may be extended to provide response estimates (Milford, 1982). Following the full-scale and laboratory observation of chimneys, van Koten (1986) has formulated an expression to predict the response amplitudes of chimneys in terms of damping, aspect ratio, Scruton number and empirical coefficients derived from observed data. Vickery and Basu (1983b) also proposed an equivalent static load model in which the force coefficient was obtained by matching the response amplitude resulting from the equivalent static load to the response defined by an acrosswind response prediction model. The acrosswind response at the critical wind speed may be estimated following a simple static analysis utilizing model properties. While the equivalent static load approaches are easyto-use and hence popular in the design specifications, their major weakness rests in their sensitivity to the choice of the critical wind speed and the selection of chimney level at which the critical wind speed will occur. Other empirical procedures reported in the literature and any recent revisions of the methods examined here have not been investigated here.

\subsection{Response Predlction Based on Aeroelastic Modeis}

The spine-type aeroelastic scale models of $130 \mathrm{~m}$ and $180 \mathrm{~m}$ prototype chimneys were tested under simulated flow conditions with smooth and optimal roughness attached. No significant difference in results was noticed for the two model surface conditions. The ineffectiveness of roughness elements in this case is attributed to inability of the boundary layer wind tunnel, at the University of Houston, used in this experiment to attain speeds high enough to reach minimum Reynolds number of 2.5 $\times 10^{4}$ necessary for the flow field to attain the characteristics of high Reynolds number flow. In the earlier experiment on a rigid cylinder it was possible to reach the target Reynolds number since the cylinder diameter was three inches, whereas in scaling of prototype chimneys, the diameter was close to one inch. These measurements also suggested that the motion of the chimney models did not introduce any modification to influence flow sensitivity to Reynolds numbers.

In view of the above results, a second phase of experiments was conducted in a wind tunnel in R.O.C. by the first author using new larger size models made out of Devcon. This study was limited only to $265 \mathrm{~m}$ and $130 \mathrm{~m}$ chimneys (Melbourne, et al., 1983 and Christensen and Askegaard, 1978). The necessary Reynolds number for the roughness to become effective were reached and the results suggested a significant modification. The damping ratio of the wind tunnel model was slightly higher than the damping reported for the full-scale chimney. This discrepency was adjusted by correcting the experimental results by an appropriate damping scaling factor. 


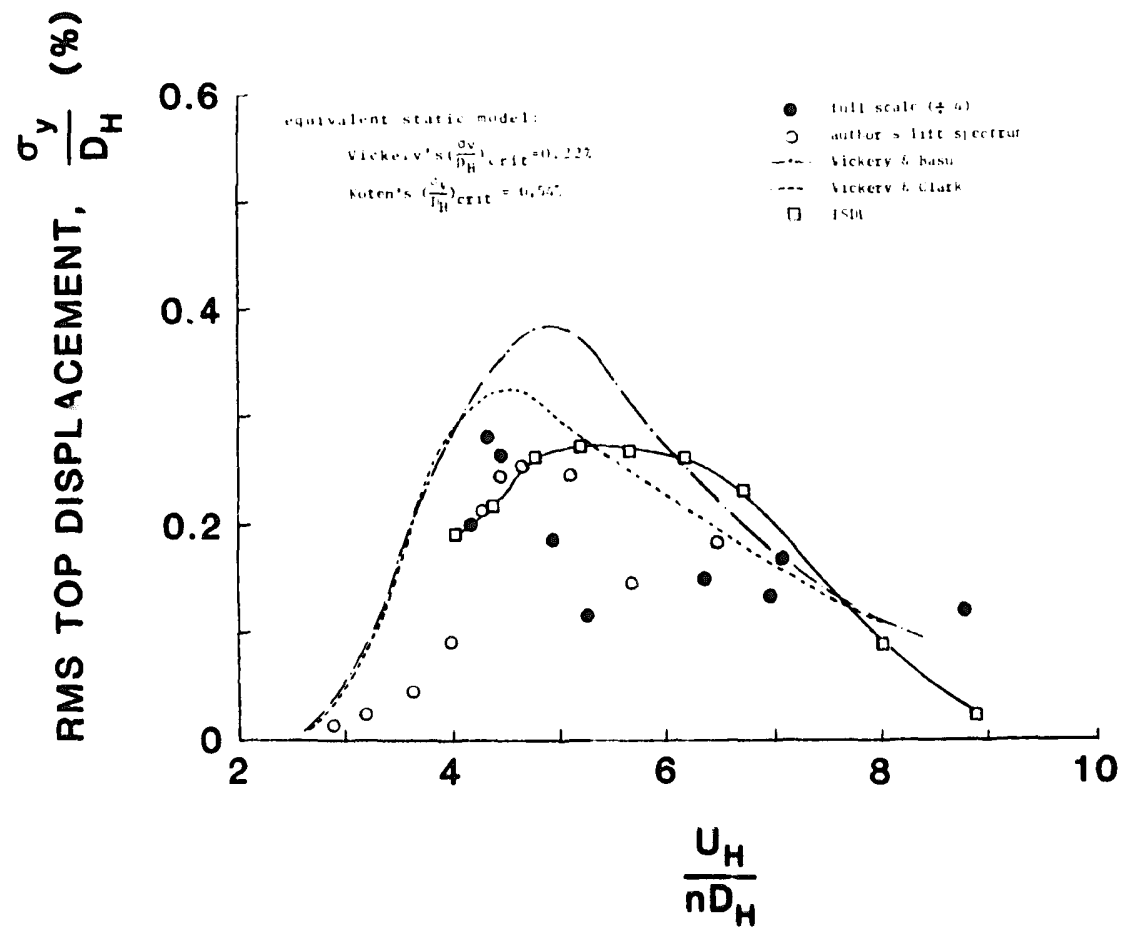

Figure 3. Comparison of predicted response with full-scale measurements of $180 \mathrm{~m}$ chimney.

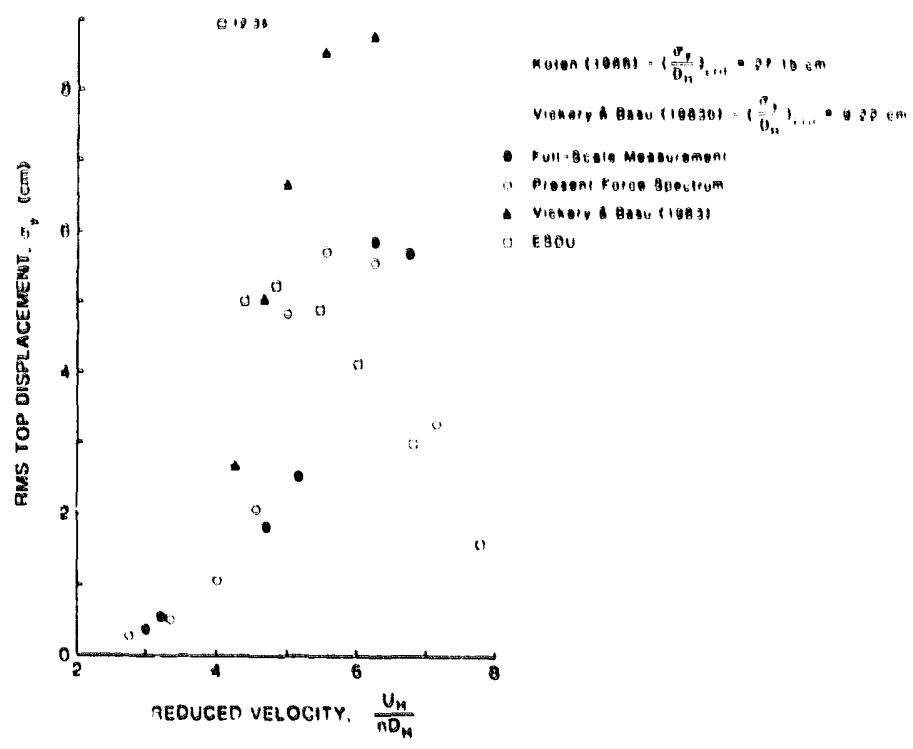

Figure 4. Comparison of predicted response with full-scale measurements of $265 \mathrm{~m}$ chimney. 


\section{RESULTS}

The response predictions based on the measured force spectra and other predictive models for $130 \mathrm{~m}, 180 \mathrm{~m}$ and $265 \mathrm{~m}$ chimneys are presented in Figs. $2-4$. It should be noted that Basu and Vickery (1982) found the originally reported response estimates of the $180 \mathrm{~m}$ chimney to be in error by a factor of four times the actual response; therefore, corrected response estimates are given in Fig. 3. The equivalent static loading approach due to van Koten (1979) produced a large margin of error, $264 \%$ and $93 \%$ for $130 \mathrm{~m}$ and $180 \mathrm{~m}$ chimneys, respectively [Figs. 2 \& 3i. This large discrepency between the predicted and observed values may be attributed to the fact that van Koten's formulation was primarily for steel stacks. Vickery and Basu's (1983b) equivalent static formulation predicted the response of $180 \mathrm{~m}$ chimney quite well with only $21 \%$ underestimation. However, for the $130 \mathrm{~m}$ chimneys, this method overestimated the full-scale acrosswind response. ESDU (1978) method provided good estimates for the $180 \mathrm{~m}$ chimney but departed significantly from the measured response of the 130m chimney. Models due to Vickery and Basu (1983a), Vickery and Clark (1972) and the experimental method utilizing simulated high Reynolds number flow provided the best comparisons with the full-scale response. These approaches also predicted the first resonant peak wind speed o: $180 \mathrm{~m}$ chimney very well but showed a rather large shift for the $130 \mathrm{~m}$ chimney.

The scatter in the predicted and observed response estimates may be attributed to the failure of the predictive models to identify the dependency of the aerodynamic pairmeters used in the model on turbulence. In recognition of this dependence of the aerodynamic parameters on the level of turbulence, measured values of these parameters at the site of $130 \mathrm{~m}$ chimney were available, thus these were incorporated in the predictive models (Christensen and Askegaard, 1978; Vickery and Basu, 1983a). The comparison of response is presented in Fig. $2 \mathrm{~b}$. The results indicate that both equivalent static methods due to van Koten and Vickery and Basu still overestimate the response at critical wind speeds by $167 \%$ and $100 \%$, respectively. The ESDU method overestimates the measured response by $78 \%$. The other two predictive models (Vickery and Basu (1983a) and Vickery and Clark, (1972)) and the present force spectrum approach provided closer agreement.

The agreement between the predictions based on the measured force spectrum and the observed rms response of the $265 \mathrm{~m}$ tall concrete chimney (Mieibourne, Cheung, Goddard, 1983) is very good, whereas Vickery and Basu's (1983a) model provides a fair agreement (Fig. 4). The quasi static approaches due to van Koten (1979) and Vickery and Basu (1983b) predicted the response to be 27.15 and 9.22 centimeter, respectively.

The spine type aeroelastic models used to predict the response of $130 \mathrm{~m}$ and $180 \mathrm{~m}$ chimneys showed a poor comparison with the full-scale data despite the introduction of optimal surface roughness. The lack of comparison may be characterized by a discrepancy in agreement between the model and full-scale strouhal number as well as the response amplitude. The primary cause for this disagreement is inability to reach minimum Reynolds number that are necessary for the discrete surface roughness to become effective $\left(R_{n}=2.5 \times 10^{4}\right.$; Kareem \& Cheng, 1984).

In view of the preceding difficulty in generating the minimum level of Reynolds numbers for simulating artificially the high Reynolds number effects, additional aeroelastic studies were conducted in a $7 \mathrm{ft} \times 10 \mathrm{ft}$ wind tunnel at the Academia Sinica, R.O.C. The models of $130 \mathrm{~m}$ and $256 \mathrm{~m}$ chimneys were made out of Devcon. The mocielling details and surrounding terrain modelling can be found in Cheng (1990). For the sake of brevity these details are not included here. 
The response of the $256 \mathrm{~m}$ chimney based on the aeroelastic model is presented in Fig. 5. The results include the response of the chimney model without any roughness (smooth), model with roughness and the results adjusted for two damping conditions, and the data from full-scale measurements. The adjustment in the damping value was made necessary due to slightly higher damping present in the wind tunnel model. The predicted response is scaled to correspond to the full-scale damping values using the dependence of response on damping as $\xi^{-1}$ and $\xi^{-1 / 2}$. These corrections correspond to harmonic and random oscillations. It is noted that the results show a good agreement for different oscillation regimes, i.e.; for non resonant conditions the damping correction for random oscillation is more appropriate, whereas, near resonant conditions the response amplitudes adjusted for harmonic oscillations provide a good comparison. Although the roughness elements help to predict quite accurately the chimney response, there remains some discrepency concerning the reduced velocity associated with the resonant response. A similar pattern was noted for the response comparison based on measured force spectra. The response prediction based on aeroelastic model of the $130 \mathrm{~m}$ chimney show a good agreement with full-scale data. Limited space here does not permit presentation of the results.

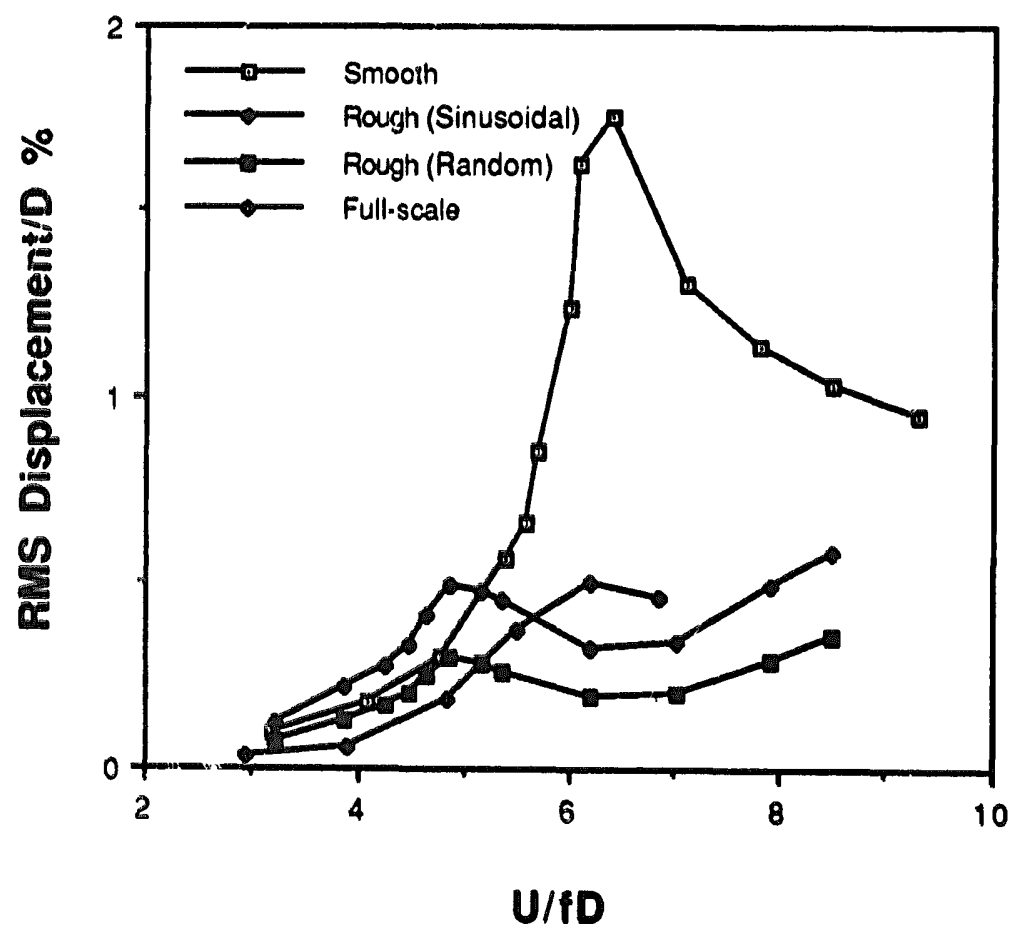

Figure 5. Comparison of wind tunnel and full-scale response of $256 \mathrm{~m}$ chimneys.

\section{CONCLUSIONS}

Two pairs of wires installed on a circuiar cylinder, the first pair to help promote transition to turbulence and the second pair to introduce separation, help to simulate 
artificially flow field features past a cylinder that represent the characteristics of high Reynolds number flows. Experiments also revealed that in order for this roughness to be effective a minirnum Reynolds number of $2.27 \times 10^{4}$ is necessary.

- The acrosswind force spectrum measured on a rigid cylinder with the above surface roughness provides response estimates of chimneys with a good agreement with the observed full-scale response.

- With an appropriate selection of the aerodynamic parameters based on the farfield turbulence at a site the predictive models provide more accurate estimates of the acrosswind response of concrete chimneys.

- The aeroelastic models of chimneys with the proposed roughness elements predict the acrosswind response of chimneys that have a good agreement with the observed full-scale response.

\section{ACKNOWLEDGEMENTS}

Financial assistance for this research was provided in part by NSF grant BCS8352223 (BCS-9096274) and a grant from R.O.C.

\section{REFERENCES}

E. Achenback, (1981) "On Vortex Shedding from Smooth and Rough Cylinders in the Range of Reynolds Number $6 \times 10^{3}$ to $5 \times 10^{6}$," J. of Fluid Mechanics, 109.

R.I. Basu and B.J. Vickery, (1982) "A Comparison of Model and Full-scale Behavior in Wind of Towers and Chimneys," Wind Tunnel Modelling for Civil Engineering Applications, Cambridge University Press.

J.P. Batham, (1973) "Pressure Distribution on Circular Cylinder at Critical Reynolds Numbers," J. of Fluid Mechanics, 57(2).

C-M Cheng, (1990) "Aerodynamic Modeling of Chimneys," Structures Congress 1990, San Francisco, ASCE.

O. Christensen and V. Askegaard, (1978) "Wind Forces on and Excitation of a 130m Concrete Chimney," J. Industrial Aerodynamics, Vol. 3, No. 2.

ESDU, (1978) "Across-flow Response due to Vortex Sheddding: Isolated Circular Cylinderical Structures in wind or gas flows," Engineering Science Data Item Number 78006.

C. Farell and O. Guven, (1976) "Mean Wind Load on Rough-Walled Cooling Towers," J. of Engr. Mech., ASCE.

S.O. Hansen, (1981) "Crosswind Vibration of a 130m Tapered Concrete Chimney," J. Wind Engineering and Industrial Aerodynamics, Vol. 8.

A. Kareem, (1981) "Discussion of Wind-Induced Lock-In Excitation of Tall Structures," by Kenny C.S. Kwok and W.H. Melbourne, Journal of the Struct. Div., ASCE, Vol. 107, ST2, Jan.; Journal of the Struct. Div., ASCE, Vol. 107, ST10, Oct.

A. Kareem, (1982) "Acrosswind Response of Buildings," J. of the Struct. Div., ASCE, Vol. 108, No. ST4.

A. Kareem, (1984) "A Model for Prediction of the Acrosswind Response of Buildings," Engineering Structures, Vol. 6, No. 2.

A. Kareem, (1987) "Wind Effects on Structures: A Probabilistic Viewpoint," Probabilistic Engineering Mechanics, 2.

A Kareem and C-M Cheng, (1984) "Acrosswind Response of Towers and Stacks of Circular Cross-Section," Department of Civil Engineering, University of Houston, 
Report No. UHCE 84-5 August.

W.H. Melbourne, J.C.K. Cheung, and C.R. Goddard, (1983) "Response to Wind Action of 265-m Mount Isa Stack," Journal of Structural Div., ASCE, Vol. 109, No. 11 , Nov.

R.V. Milford, (1982) "Appraisal of Methods for Predicting the Acrosswind Response of Chimneys," Proc. Inst. Civ. Engrs., Part 2, Vol. 73.

F.P. Muller and H. Niesser, (1976) "Measurements of Wind Induced Vibrations on a Concrete Chimney," J. of Industrial Aerodynamics, Vol. 1.

Y. Nakamura and Y. Tomonrai, (1982) "The Effects of Surface Roughness on the Flow Past Circular Cylinders at High Reynolds Numbers," J. Fluid Mechanics, 123.

A. Naumann and H. Ouadflieg, (1972) "Vortex Generation on a Cylindrical Building and its Simulation in Wind Tunnel," Flow Induced Structural Vibrations, IUTAMIAHR Symposium, Karlsruhe.

J.W.G. van Nunen, (1972), "Pressures and Forces on a Circular Cylinder in a Cross Flow at High Reynolds Number," Flow Induced Vibrations, IUTAM-IAHR Symposium, Karlsruhe.

H. Ruscheweyh and G. itirsch, (1975), "Full-Scale Measurements of Dynamic Response of Tower Shaped Structures," 4th International Conf. on Wind Effects on Buildings and Structures, London.

H. Schlichting, (1979), Boundary Layer Theory, McGraw Hill.

E. Simiu and R.H. Scanlan, (1986) Wind Effects on Structures, Second Edition, Wiley Interscience.

E. Szechenyi, (1975) "Subcritical Reynolds Number Simulation for Two-Dimensional Flow Over A Circular Cylinder," J. of Fluid Mechanics, 70.

H. van Koten, (1986) "Crosswind Movements of Chimneys," Heron, Vol. 31, No. 1, Dept. of Civil Engineering, Delft University of Tech and TNO.

B.J. Vickery and R.I. Basu, (1983a) "Across-wind Vibrations of Structures of Circular Cross-Section. Part I. Development of a Mathematical Model for Two-dimensional Conditions," J. Wind Engineering and Industrial Aerodynamics, Vol. 12, May.

B.J. Vickery and R.I. Basu, (1983b) "Simplified Approaches to the Evaluation of the Across-Wind Response of Chimney," Journal of Wind Engineering and Industrial Aerodynamics, Vol. 14

B.J. Vickery and A.W. Clark, (1972) "Lift or Crosswind Response of Tapered Stacks," J. of Struct. Div., ASCE, Vol. 98, No. ST1. 